\title{
Erratum to: Radiation-induced changes in peripheral nerve by stereotactic radiosurgery: a study on the sciatic nerve of rabbit
}

\author{
Zhixiong Lin • Vincent W. C. Wu $\cdot$ Wenchui Ju • \\ Yoshiya Yamada $\cdot$ Longhua Chen
}

Published online: 21 September 2010

(C) Springer Science+Business Media, LLC. 2010

\section{Erratum to: J Neurooncol}

\section{DOI 10.1007/s11060-010-0309-3}

The first author's and the last author's main affiliation was not included in the original publication. This erratum shows their affiliations correctly.

The online version of the original article can be found under doi: 10.1007/s11060-010-0309-3.

Z. Lin · L. Chen

Department of Radiation Oncology, Nanfang Hospital, Southern

Medical University, Nanfang, China

Z. Lin · W. Ju · L. Chen

Department of Radiation Oncology, Cancer Hospital, Shantou

University Medical College, Shantou, China

V. W. C. Wu ( $\square)$

Department of Health Technology \& Informatics, Hong Kong

Polytechnic University, Hung Hom, Kowloon, Hong Kong

e-mail: htvinwu@polyu.edu.hk

Y. Yamada

Department of Radiation Oncology, Memorial Sloan Kettering

Cancer Center, New York, USA 